\title{
ASYMPTOTIC BEHAVIOR OF SOLUTIONS TO ELLIPTIC AND PARABOLIC EQUATIONS WITH UNBOUNDED COEFFICIENTS OF THE SECOND ORDER IN UNBOUNDED DOMAINS
}

\author{
HIDEO KOZONO, YUTAKA TERASAWA AND YUTA WAKASUGI \\ Dedicated to Professor Matthias Hieber on the occasion of his 60th birthday
}

\begin{abstract}
We study an asymptotic behavior of solutions to elliptic equations of the second order in a two dimensional exterior domain. Under the assumption that the solution belongs to $L^{q}$ with $q \in[2, \infty)$, we prove a pointwise asymptotic estimate of the solution at the spatial infinity in terms of the behavior of the coefficients. As a corollary, we obtain the Liouville-type theorem in the case when the coefficients may grow at the spacial infinity. We also study a corresponding parabolic problem in the $n$-dimensional whole space and discuss the energy identity for solutions in $L^{q}$. As a corollary we show also the Liouville-type theorem for both forward and ancient solutions.
\end{abstract}

\section{INTRODUCTION}

1

We consider an elliptic differential equation of the second order with the divergence form such as

$$
-\sum_{i, j=1}^{2} \partial_{i}\left(a_{i j}(x) \partial_{j} u\right)+\mathbf{b}(x) \cdot \nabla u+c(x) u=0, \quad x \in \Omega
$$

where $\Omega$ is the whole plane $\mathbb{R}^{2}$ or an exterior domain $\Omega={\overline{B_{r_{0}}(0)}}^{c}=\left\{x \in \mathbb{R}^{2} ;|x|=\right.$ $\left.r \geq r_{0}\right\}$. Our aim is to clarify how the asymptotic behavior of the coefficients $a_{i j}(x)$ and $\mathbf{b}(x)$, in particular, their growth conditions at infinity, has an influence to the asymptotic behavior of solution $u(x)$ of (1.1) as $|x| \rightarrow \infty$. Our study is motivated by investigation of the asymptotic behavior of solutions to the stationary NavierStokes equations

$$
\left\{\begin{array}{l}
-\Delta v+(v \cdot \nabla) v+\nabla p=0, \quad x \in \Omega . \\
\operatorname{div} v=0
\end{array}\right.
$$

By the pioneer work of Leray [15, the existence of solutions $(v, p)$ of (1.2) with the finite Dirichlet integral

$$
\int_{\Omega}|\nabla v(x)|^{2} d x<\infty
$$

had been proved. Then, Gilbarg-Weinberger [8, Amick [1, and Korobkov-PileckasRusso [11, 12] studied the asymptotic behavior of solutions satisfying (1.3). They

Key words and phrases. elliptic and parabolic equations of second order; asymptotic behavior. 2010 Mathematics Subject Classification. 35J15, 35K10, 35B53 
proved that the solution $v$ to (1.2) - (1.3) converges to a constant vector $v_{\infty}$ uniformly at infinity, i.e.,

$$
\lim _{r \rightarrow \infty} \sup _{\theta \in[0,2 \pi]}\left|v(r, \theta)-v_{\infty}\right|=0
$$

where $(r, \theta)$ denotes the polar coordinates. A basic approach to the analysis of (1.2) is to handle the vorticity $\omega=\operatorname{rot} v=\partial_{x_{1}} v_{2}-\partial_{x_{2}} v_{1}$ which satisfies the equation

$$
-\Delta \omega+v \cdot \nabla \omega=0 .
$$

In our previous result [14, we studied the asymptotic behavior of solutions $\omega$ to (1.4) with the finite generalized Dirichlet integral

$$
\int_{\Omega}|\nabla v(x)|^{q} d x<\infty
$$

for some $q \in(2, \infty)$. Note that (1.5) implies $\omega \in L^{q}(\Omega)$. Indeed, it is proved in [14] that the vorticity $\omega$ and the gradient $\nabla v$ of the velocity behave like

$$
|\omega(r, \theta)|=o\left(r^{-\left(\frac{1}{q}+\frac{1}{q^{2}}\right)}\right), \quad|\nabla v(r, \theta)|=o\left(r^{-\left(\frac{1}{q}+\frac{1}{q^{2}}\right)} \log r\right) \quad \text { as } r \rightarrow \infty,
$$

respectively. The crucial point is to regard the velocity $v(x)$ as a given coefficient in the equation (1.4) and to analyze how the asymptotic behavior of $v(x)$ does affect that of $\omega$. In this respect, the problem (1.1) may be regarded as a generalization of (1.4).

In this paper, we generalize the result of 14 to the elliptic equation (1.1), and prove the asymptotic behavior of solutions at the spatial infinity under the assumption that $u \in L^{q}(\Omega)$ with some $q \in[2, \infty)$. In particular, we are interested in the case when the coefficients $a_{i j}, b$ may grow at spatial infinity.

Our precise assumptions and results are the following.

\section{Assumptions on the coefficients for the elliptic problem (1.1)}

(e-i) $a_{i j} \in C^{1}(\Omega), a_{i j}=a_{j i}$ for $i, j=1,2$ and

$$
\sum_{i, j=1}^{2} a_{i j}(x) \xi_{i} \xi_{j} \geq \lambda|\xi|^{2} \quad\left(\xi \in \mathbb{R}^{2}, x \in \Omega\right)
$$

with some $\lambda>0$. The growth condition

$$
\left|a_{i j}(x)\right|=O\left(|x|^{\alpha}\right), \quad\left|\partial_{i} a_{i j}(x)\right|=O\left(|x|^{\alpha-1}\right) \quad(|x| \rightarrow \infty)
$$

is satisfied for $i, j=1,2$ with some $\alpha \in[0,2]$.

(e-ii) $\mathbf{b}(x)=\left(b_{1}(x), b_{2}(x)\right) \in C^{1}(\Omega)$ satisfies

$$
\mathbf{b}(x)=O\left(|x|^{\beta}\right) \quad(|x| \rightarrow \infty)
$$

with some $\beta \leq 1$.

(e-iii) $c(x)$ is measurable and nonnegative.

(e-iv) Either following condition (1) or (2) holds:

(1) $\operatorname{div} \mathbf{b}(x) \leq 2 c(x)$,

(2) $|\operatorname{div} \mathbf{b}(x)|=O\left(|x|^{\beta-1}\right) \quad(|x| \rightarrow \infty)$.

Our first result on the elliptic equation (1.1) now reads 
Theorem 1.1. Let the assumptions (e-i)-(e-iv) above hold. Suppose that that $u \in$ $C^{2}(\Omega)$ satisfies (1.1) in $\Omega$ and that $u \in L^{q}(\Omega)$ with some $q \in[2, \infty)$. Then, we have that

$$
\sup _{0 \leq \theta \leq 2 \pi}|u(r, \theta)|=o\left(r^{-\frac{1}{q}\left(1+\frac{\gamma}{2}\right)}\right) \quad \text { as } r \rightarrow \infty,
$$

where $\gamma=\min \{1-\beta, 2-\alpha\}$.

Remark 1.1. When $\alpha=0$ and $\beta \leq-1$, we have

$$
\sup _{0 \leq \theta \leq 2 \pi}|u(r, \theta)|=o\left(r^{-\frac{2}{q}}\right) \quad \text { as } r \rightarrow \infty,
$$

which exhibits the correspondence to the condition $u \in L^{q}(\Omega)$.

As a corollary of the above theorem, we have the following Liouville-type result.

Corollary 1.2. Assume (e-i)-(e-iv). Let $\Omega=\mathbb{R}^{2}$ and let $u \in C^{2}\left(\mathbb{R}^{2}\right)$ be a solution to (1.1) satisfying $u \in L^{q}\left(\mathbb{R}^{2}\right)$ with some $q \in[2, \infty)$. Then, it holds that $u \equiv 0$ on $\mathbb{R}^{2}$.

Remark 1.2. The above corollary is sharp in the sense that if $q=\infty$, then there exists a solution $u$ of (1.1) which is not a constant. Indeed, let $a_{i j}(x)=\delta_{i j}$, $\mathbf{b}(x)=\left(-x_{1}, x_{2}\right)$ (namely, $\left.\beta=1\right)$, and $c(x) \equiv 0$. Consider $u=u\left(x_{1}, x_{2}\right)=f\left(x_{1}\right)$ with

$$
f(\tau)=\int_{0}^{\tau} e^{-s^{2} / 2} d s, \quad \tau \in \mathbb{R} .
$$

It is easy to see that $u \in L^{\infty}\left(\mathbb{R}^{2}\right)$ with $-\Delta u+\mathbf{b}(x) \cdot \nabla u=0$ in $\mathbb{R}^{2}$. Obviously, $u$ is not a constant.

Remark 1.3. For the elliptic equation (1.1) with $\Omega=\mathbb{R}^{n}$ and $n \geq 1$, the result by Seregin-Silvestre-Šverák-Zlatoš [17, Theorem 1.2] implies a Liouville-type theorem under the conditions that $a_{i j}$ is bounded, $\mathbf{b} \in B M O^{-1}$ with $\operatorname{div} \mathbf{b}=0$, and that $c \equiv$ 0 , namely, every bounded solutions are constants. Compared with their theorem, our result allows the coefficients $a_{i j}, b$ to grow at spacial infinity. On the other hand, we impose on the stronger assumption on the solution such as $u \in L^{q}(\Omega)$ with some $q \in[2, \infty)$

Remark 1.4. The above corollary may be regarded as a generalization of 14 , Corollary 1.2], which states that every smooth solution $v$ of $(1.2)$ in $\mathbb{R}^{2}$ satisfying the condition $\nabla v \in L^{q}\left(\mathbb{R}^{2}\right)$ for some $q \in(2, \infty)$ must be a constant vector. Recently, Liouville-type theorems of the stationary Navier-Stokes equations are fully studied, and we refer the reader to $2,4,5,6,9,13,16$, and the references therein.

The proofs of Theorem 1.1 and Corollary 1.2 are given in the next section. Our approach is based on that of Gilbarg and Weinberger [8] and its generalization introduced in 14. We first show a certain elliptic estimate of the solution $u$ by the energy method (Lemma 2.1). Then, combining it with the integral mean value theorem for the radial variable $r$ and the fundamental theorem of calculus for the angular variable $\theta$, we derive a pointwise decay estimate of the solution along with a special sequence $\left\{r_{n}\right\}_{n=1}^{\infty}$ of the radial variable satisfying $\lim _{n \rightarrow \infty} r_{n}=\infty$. Finally, applying the maximum principle in the annular domains between $r=r_{n-1}$ and $r=r_{n}$, we have the desired uniform decay like (1.6) (Lemma 2.2). 
Furthermore, our approach is also applicable to parabolic problems. We discuss energy estimates of solutions to the corresponding parabolic equation in the $n$ dimensional whole space $\mathbb{R}^{n}$;

$$
\partial_{t} u-\sum_{i, j=1}^{n} \partial_{i}\left(a_{i j}(x, t) \partial_{j} u\right)+\mathbf{b}(x, t) \cdot \nabla u+c(x, t) u=0, \quad x \in \mathbb{R}^{n}, t \in I,
$$

where $I \subset \mathbb{R}$ is an interval. We impose similar assumptions on the coefficients $a_{i j}(x, t), \mathbf{b}(x, t)$ and $c(x, t)$, on the premise that they are measurable functions on $\mathbb{R}^{n} \times I$ :

\section{Assumptions on the coefficients for the parabolic problem (1.7)}

(p-i) $a_{i j} \in C^{1,0}\left(\mathbb{R}^{n} \times I\right), a_{i j}=a_{j i}$ for $i, j=1, \cdots, n$ and

$$
\sum_{i, j=1}^{n} a_{i j}(x, t) \xi_{i} \xi_{j} \geq \lambda|\xi|^{2} \quad\left(\xi \in \mathbb{R}^{n}, x \in \mathbb{R}^{n}, t \in I\right)
$$

with some $\lambda>0$. The growth condition

$$
\left|a_{i j}(x, t)\right|=O\left(|x|^{2}\right), \quad\left|\partial_{i} a_{i j}(x, t)\right|=O(|x|) \quad(|x| \rightarrow \infty)
$$

holds locally uniformly in $t \in I$.

$\left(\mathrm{p}\right.$-ii) $\mathbf{b}(x, t)=\left(b_{1}(x, t), \ldots, b_{n}(x, t)\right) \in C^{1,0}\left(\mathbb{R}^{n} \times I\right)$ satisfies

$$
\mathbf{b}(x, t)=O(|x|) \quad(|x| \rightarrow \infty)
$$

locally uniformly in $t \in I$.

(p-iii) $c(x, t)$ is nonnegative, and $\operatorname{div} \mathbf{b}(x, t)=\sum_{j=1}^{n} \partial_{x_{j}} b_{j}(x, t) \leq 2 c(x, t)$ holds for all $(x, t) \in \mathbb{R}^{n} \times I$.

Under these assumptions, we show the following energy identity for solutions belonging to $L^{q}\left(\mathbb{R}^{n} \times I\right)$.

Theorem 1.3. Assume (p-i) and (p-ii). Let $u \in C^{2,1}\left(\mathbb{R}^{n} \times I\right)$ be a solution to (1.7) satisfying $u \in L^{q}\left(\mathbb{R}^{n} \times I\right)$ with some $q \in[2, \infty)$. Then, we have the energy identity

$$
\begin{aligned}
& \int_{\mathbb{R}^{n}}|u(x, t)|^{q} d x+q(q-1) \int_{s}^{t} \int_{\mathbb{R}^{n}}|u(x, \tau)|^{q-2} \sum_{i, j=1}^{n} a_{i j}(x, \tau) \partial_{i} u(x, \tau) \partial_{j} u(x, \tau) d x d \tau \\
& \quad+\int_{s}^{t} \int_{\mathbb{R}^{n}}(-\operatorname{div} \mathbf{b}(x, \tau)+q c(x, \tau))|u(x, \tau)|^{q} d x d \tau \\
& =\int_{\mathbb{R}^{n}}|u(x, s)|^{q} d x
\end{aligned}
$$

for all $t, s \in I$ such that $s \leq t$.

Remark 1.5. In Theorem 1.3, we do not need the assumption (p-iii).

By Theorem 1.3. we have the following Liouville-type results on solutions of the Cauchy problem of (1.7) and on ancient solutions of (1.7).

Corollary 1.4. In addition to (p-i) and (p-ii), assume that (p-iii) holds.

(i) Let $u \in C^{2}\left(\mathbb{R}^{n} \times[0, T)\right)$ be a solution of (1.7) satisfying $u \in L^{q}\left(\mathbb{R}^{n} \times[0, T)\right)$ with some $q \in[2, \infty)$. Moreover, we assume that $u(x, 0) \equiv 0$ on $\mathbb{R}^{n}$. Then, we have $u \equiv 0$ on $\mathbb{R}^{n} \times[0, T)$. 
(ii) Let $u \in C^{2}\left(\mathbb{R}^{n} \times(-\infty, 0)\right)$ be an ancient solution of (1.7) satisfying $u \in L^{q}\left(\mathbb{R}^{n} \times\right.$ $(-\infty, 0))$ with some $q \in[2, \infty)$. Then, we have $u \equiv 0$ on $\mathbb{R}^{n} \times(-\infty, 0)$.

Remark 1.6. For the heat equation $\partial_{t} v-\Delta v=0$ on a complete noncompact Reimannian manifold with the nonnegative Ricci curvature, Souplet-Zhang [18] proved that any positive ancient (or entire) solution $u$ having the bound

$$
u(x, t)=O\left(e^{o(d(x)+\sqrt{t})}\right) \quad \text { as } d(x) \rightarrow \infty
$$

must be a constant, where $d(x)$ is the distance from a base point. They also proved that any ancient (or entire) solution $u$ having the bound

$$
u(x, t)=o(d(x)+\sqrt{t}) \quad \text { as } d(x) \rightarrow \infty
$$

must be a constant. Compared with their result, we are able to treat more general time-dependent coefficients which may grow at the spatial infinity. On the other hand, we impose solutions $u$ on the stronger assumption that $u \in L^{q}\left(\mathbb{R}^{n} \times I\right)$ with some $q \in[2, \infty)$.

Remark 1.7. The Liouville-type theorem for the non-stationary Navier-Stokes equations

$$
\left\{\begin{array}{l}
\partial_{t} v-\Delta v+(v \cdot \nabla) v+\nabla p=0, \quad(x, t) \in \mathbb{R}^{n} \times I \\
\operatorname{div} v=0
\end{array}\right.
$$

has been fully studied, where $I=(0, T)$ or $I=(-\infty, 0)$. We refer the reader to [10, 3, 7] and the references therein.

\section{Proof of Theorem 1.1}

In what follows, we shall denote by $C$ various constants which may change from line to line. In particular, we denote by $C=C(*, \ldots, *)$ constants depending only on the quantities appearing in parentheses.

Lemma 2.1. Under the assumptions on Theorem 1.1, for every $r_{1}>r_{0}$, we have

$$
\int_{r \geq r_{1}} r^{\gamma}|u|^{q-2}|\nabla u|^{2} d x \leq C\left(q, r_{1}\right) \int_{\Omega}|u|^{q} d x
$$

where $\gamma=\min \{1-\beta, 2-\alpha\}$.

Proof. Let $\eta=\eta(r) \in C_{0}^{\infty}(\Omega)$ and let $h=h(u) \in C^{1}(\mathbb{R})$ be a piecewise $C^{2}$ function specified later. We start with the following identity:

$$
\begin{aligned}
& -\sum_{i=1}^{2} \partial_{i}\left[\eta(r) \sum_{j=1}^{2} a_{i j} \partial_{j}(h(u))-\sum_{j=1}^{2} a_{i j}\left(\partial_{j} \eta\right) h(u)-\eta(r) h(u) b_{i}(x)\right] \\
& =-\eta(r) h^{\prime \prime}(u)\left(\sum_{i, j=1}^{2} a_{i j} \partial_{i} u \partial_{j} u\right) \\
& +h(u)\left[\sum_{i, j=1}^{2} \partial_{j}\left(a_{i j} \partial_{i} \eta\right)+\mathbf{b}(x) \cdot \nabla \eta(r)+\eta(r) \operatorname{div} \mathbf{b}\right] \\
& -\eta(r) h^{\prime}(u)\left[\sum_{i, j=1}^{2} \partial_{j}\left(a_{i j} \partial_{i} u\right)-\mathbf{b} \cdot \nabla u\right]
\end{aligned}
$$


Since $u$ satisfies the equation (1.1), integration of the above identity over $\Omega$ yields

$$
\begin{aligned}
\int_{\Omega} \eta(r) h^{\prime \prime}(u)\left(\sum_{i, j=1}^{2} a_{i j} \partial_{i} u \partial_{j} u\right) d x= & \int_{\Omega} h(u)\left[\sum_{i, j=1}^{2} \partial_{j}\left(a_{i j} \partial_{i} \eta\right)+\mathbf{b}(x) \cdot \nabla \eta(r)\right] d x \\
& +\int_{\Omega} \eta(r)\left(h(u) \operatorname{div} \mathbf{b}(x)-h^{\prime}(u) c(x) u\right) d x .
\end{aligned}
$$

Let $r_{1}>r_{0}$ and let $\xi_{1}=\xi_{1}(r) \in C^{\infty}(\Omega)$ be nonnegative, monotone increasing in $r$, and satisfy $\xi(r)=1$ for $r \geq r_{1}$ and $\xi(r)=0$ for $r \leq\left(r_{0}+r_{1}\right) / 2$. Let $\xi_{2}=$ $\xi_{2}(r) \in C_{0}^{\infty}\left(B_{1}(0)\right)$ be nonnegative, monotone decreasing, and satisfy $\xi_{2}(r)=1$ for $r \leq 1 / 2$. We choose the cut-off function $\eta(r)$ as

$$
\eta(r)=r^{\gamma} \xi_{1}(r) \xi_{2}\left(\frac{r}{R}\right),
$$

with the parameter $R \geq 1$, where $\gamma=\min \{1-\beta, 2-\alpha\}$. Then, we have $|\nabla \eta(r)| \leq$ $C r^{\gamma-1},\left|\partial_{i} \partial_{j} \eta(r)\right| \leq C r^{\gamma-2}$. Now, we take $h(u)=|u|^{q}$. Then, it holds that $h^{\prime}(u)=$ $q|u|^{q-2} u$ and $h^{\prime \prime}(u)=q(q-1)|u|^{q-2}$. Therefore, we obtain

$$
\begin{aligned}
& q(q-1) \int_{\Omega} \eta(r)|u|^{q-2}\left(\sum_{i, j=1}^{2} a_{i j}(x) \partial_{i} u \partial_{j} u\right) d x \\
& =\int_{\Omega}|u|^{q}\left[\sum_{i, j=1}^{2} \partial_{i}\left(a_{i j}(x) \partial_{j} \eta\right)+\mathbf{b}(x) \cdot \nabla \eta\right] d x \\
& +\int_{\Omega} \eta(\operatorname{div} \mathbf{b}(x)-q c(x))|u|^{q} d x .
\end{aligned}
$$

By the assumptions (e-i) and (e-ii), the estimates

$$
\begin{aligned}
& |\mathbf{b}(x) \cdot \nabla \eta(r)| \leq C, \\
& \left|a_{i j}(x) \partial_{i} \partial_{j} \eta(r)\right| \leq C, \quad\left|\partial_{i} a_{i j}(x) \partial_{j} \eta(r)\right| \leq C, \quad i, j=1,2
\end{aligned}
$$

hold, and hence the first term of RHS of (2.1) is estimated by $C \int_{\Omega}|u|^{q} d x$. Furthermore, since $c(x) \geq 0$, implied by the assumption (e-iii), we have by (e-iv) that $\operatorname{div} \mathbf{b}(x)-q c(x) \leq 0$ or $|\eta \operatorname{div} \mathbf{b}| \leq C$, and hence,

$$
\int_{\Omega} \eta(\operatorname{div} \mathbf{b}(x)-q c(x))|u|^{q} d x \leq C \int_{\Omega}|u|^{q} d x
$$

holds in both cases. Thus, we obtain from the above estimates and the assumption (e-i) that

$$
\int_{r_{1} \leq r \leq R / 2} r^{\gamma}|u|^{q-2}|\nabla u|^{2} d x \leq C \int_{\Omega}|u|^{q} d x .
$$

Letting $R \rightarrow \infty$, we conclude

$$
\int_{r \geq r_{1}} r^{\gamma}|u|^{q-2}|\nabla u|^{2} d x \leq C \int_{\Omega}|u|^{q} d x
$$

This completes the proof of Lemma 2.1

Lemma 2.2. Under the assumptions on Theorem 1.1, we have

$$
\lim _{r \rightarrow \infty} r^{1+\frac{\gamma}{2}} \sup _{\theta \in[0,2 \pi]}|u(r, \theta)|^{q}=0 .
$$


Proof. For each sufficiently large integer $n$, let us introduce the quantity

$$
A_{n}=\int_{2^{n}}^{2^{n+1}} \frac{d r}{r} \int_{0}^{2 \pi}|u|^{q-2}\left(r^{2}|u|^{2}+r^{1+\frac{\gamma}{2}}|u|\left|\partial_{\theta} u\right|\right) d \theta .
$$

Since $\left|\partial_{\theta} u\right| \leq r|\nabla u|$, we have by Lemma 2.1 and the the Schwarz inequality that

$$
A_{n} \leq C \int_{2^{n}<r<2^{n+1}}\left(|u|^{q}+r^{\gamma}|u|^{q-2}|\nabla u|^{2}\right) d x .
$$

On the other hand, by the mean value theorem for integration, there exists $r_{n} \in$ $\left(2^{n}, 2^{n+1}\right)$ such that

$$
A_{n}=\log 2 \int_{0}^{2 \pi}\left|u\left(r_{n}, \theta\right)\right|^{q-2}\left(r_{n}^{2}\left|u\left(r_{n}, \theta\right)\right|^{2}+r_{n}^{1+\frac{\gamma}{2}}\left|u\left(r_{n}, \theta\right)\right|\left|\partial_{\theta} u\left(r_{n}, \theta\right)\right|\right) d \theta .
$$

Next, we estimate

$$
\begin{aligned}
\left|u\left(r_{n}, \theta\right)\right|^{q}-\left|u\left(r_{n}, \varphi\right)\right|^{q} & \leq\left.\left|\int_{\varphi}^{\theta} \partial_{\psi}\right| u\left(r_{n}, \psi\right)\right|^{q} d \psi \mid \\
& \leq \int_{0}^{2 \pi} q\left|u\left(r_{n}, \psi\right)\right|^{q-1}\left|\partial_{\theta} u\left(r_{n}, \psi\right)\right| d \psi .
\end{aligned}
$$

Integrating the above for $\varphi \in[0,2 \pi]$, we infer

$$
\left|u\left(r_{n}, \theta\right)\right|^{q} \leq C \int_{0}^{2 \pi}\left|u\left(r_{n}, \varphi\right)\right|^{q} d \varphi+C \int_{0}^{2 \pi} q\left|u\left(r_{n}, \psi\right)\right|^{q-1}\left|\partial_{\theta} u\left(r_{n}, \psi\right)\right| d \psi .
$$

Multiplying both sides of this estimate by $r_{n}^{1+\frac{\gamma}{2}}$ and then noting $1+\frac{\gamma}{2} \leq 2$, we have that

$$
\begin{aligned}
r_{n}^{1+\frac{\gamma}{2}}\left|u\left(r_{n}, \theta\right)\right|^{q} \leq & C r_{n}^{1+\frac{\gamma}{2}} \int_{0}^{2 \pi}\left|u\left(r_{n}, \varphi\right)\right|^{q} d \varphi \\
& +C r_{n}^{1+\frac{\gamma}{2}} \int_{0}^{2 \pi} q\left|u\left(r_{n}, \psi\right)\right|^{q-1}\left|\partial_{\theta} u\left(r_{n}, \psi\right)\right| d \psi \\
\leq & C A_{n},
\end{aligned}
$$

Consequently, we obtain

$$
r_{n}^{1+\frac{\gamma}{2}}\left|u\left(r_{n}, \theta\right)\right|^{q} \leq \int_{r>2^{n}}\left(|u|^{q}+r^{\gamma}|u|^{q-2}|\nabla u|^{2}\right) d x .
$$

Since the right-hand side of the above inequality tends to zero as $n \rightarrow \infty$, implied by Lemma 2.1, we have that

$$
\lim _{n \rightarrow \infty} r_{n}^{1+\frac{\gamma}{2}} \sup _{\theta \in[0,2 \pi]}\left|u\left(r_{n}, \theta\right)\right|^{q}=0 .
$$

Finally, since the solution $u$ of (1.1) satisfies the maximum principle and since $r_{n+1} \leq 4 r_{n}$, we estimate for $r \in\left(r_{n}, r_{n+1}\right)$ that

$$
\begin{aligned}
& r^{1+\frac{\gamma}{2}} \sup _{\theta \in[0,2 \pi]}|u(r, \theta)|^{q} \\
& \leq r_{n+1}^{1+\frac{\gamma}{2}} \max \left\{\sup _{\theta \in[0,2 \pi]}\left|u\left(r_{n}, \theta\right)\right|^{q}, \sup _{\theta \in[0,2 \pi]}\left|u\left(r_{n+1}, \theta\right)\right|^{q}\right\} \\
& \leq \max \left\{16 r_{n}^{1+\frac{\gamma}{2}} \sup _{\theta \in[0,2 \pi]}\left|u\left(r_{n}, \theta\right)\right|^{q}, r_{n+1}^{1+\frac{\gamma}{2}} \sup _{\theta \in[0,2 \pi]}\left|u\left(r_{n+1}, \theta\right)\right|^{q}\right\},
\end{aligned}
$$


which yields with the aid of (2.2) that

$$
\lim _{r \rightarrow \infty} r^{1+\frac{\gamma}{2}} \sup _{\theta \in[0,2 \pi]}|u(r, \theta)|^{q}=0 .
$$

This completes the proof of Lemma 2.2 and whence Theorem 1.3

Proof of Corollary 1.2. By the assumption (e-i), the equation (1.1) has the maximum principle. Combining this with the asymptotic behavior from Theorem 1.1 we have $u \equiv 0$.

\section{Proof of Theorem 1.3}

Let $h(u)=|u|^{q}$. We take a nonnegative function $\psi \in C_{0}^{\infty}\left(\mathbb{R}^{n}\right)$ such that

$$
\psi(x)= \begin{cases}1 & (|x| \leq 1) \\ 0 & (|x| \geq 2)\end{cases}
$$

and with the parameter $R>0$ we define

$$
\psi_{R}(x)=\psi\left(\frac{x}{R}\right) .
$$

Similarly to the previous section, by a direct computation we have

$$
\begin{aligned}
& -\sum_{i=1}^{n} \partial_{i}\left[\psi_{R} \sum_{i=j}^{n} a_{i j} \partial_{j}(h(u))-\sum_{j=1}^{n} a_{i j}\left(\partial_{j} \psi_{R}\right) h(u)-\psi_{R} h(u) b_{i}\right] \\
& =-\psi_{R} h^{\prime \prime}(u)\left(\sum_{i, j=1}^{n} a_{i j} \partial_{i} u \partial_{j} u\right) \\
& +h(u)\left[\sum_{i, j=1}^{n} \partial_{i}\left(a_{i j} \partial_{j} \psi_{R}\right)+\mathbf{b} \cdot \nabla \psi_{R}+\psi_{R} \operatorname{div} \mathbf{b}\right] \\
& -\psi_{R} h^{\prime}(u)\left[\sum_{i, j=1}^{n} \partial_{i}\left(a_{i j} \partial_{j} u\right)-\mathbf{b} \cdot \nabla u\right]
\end{aligned}
$$

Using the equation (1.7) and the identity $h^{\prime}(u) \partial_{t} u=\partial_{t}(h(u))$, we integrate the above identity over $\mathbb{R}^{n}$ to obtain

$$
\begin{aligned}
& \frac{d}{d t} \int_{\mathbb{R}^{n}} \psi_{R} h(u) d x+\int_{\mathbb{R}^{n}} \psi_{R} h^{\prime \prime}(u)\left(\sum_{i, j=1}^{2} a_{i j} \partial_{i} u \partial_{j} u\right) d x \\
& =\int_{\mathbb{R}^{n}} h(u)\left[\sum_{i, j=1}^{n} \partial_{i}\left(a_{i j} \partial_{j} \psi_{R}\right)+\mathbf{b} \cdot \nabla \psi_{R}\right] d x+\int_{\mathbb{R}^{n}} \psi_{R}\left(h(u) \operatorname{div} \mathbf{b}-c h^{\prime}(u) u\right) d x .
\end{aligned}
$$


Furthermore, since $h^{\prime}(u)=q|u|^{q-2} u$ and $h^{\prime \prime}(u)=q(q-1)|u|^{q-2}$, we integrate the above identity over $[s, t]$ to obtain

$$
\begin{aligned}
& \int_{\mathbb{R}^{n}} \psi_{R} h(u(t)) d x+q(q-1) \int_{s}^{t} \int_{\mathbb{R}^{n}} \psi_{R}|u|^{q-2} \sum_{i, j=1}^{n} a_{i j} \partial_{i} u \partial_{j} u d x d \tau \\
& \quad+\int_{s}^{t} \int_{\mathbb{R}^{n}} \psi_{R}(-\operatorname{div} \mathbf{b}+q c)|u|^{q} d x d \tau \\
& =\int_{\mathbb{R}^{n}} \psi_{R} h(u(s)) d x \\
& \quad+\int_{s}^{t} \int_{\mathbb{R}^{n}} h(u)\left[\sum_{i, j=1}^{n} \partial_{j}\left(a_{i j} \partial_{i} \psi_{R}\right)+\mathbf{b} \cdot \nabla \psi_{R}\right] d x d \tau .
\end{aligned}
$$

Let us estimate the right-hand side. Using the assumptions ( $\mathrm{p}-\mathrm{i})$ and (p-ii), and then applying the Lebesgue dominated convergence theorem, we have that

$$
\begin{aligned}
& \int_{s}^{t} \int_{\mathbb{R}^{n}} h(u) \sum_{i, j=1}^{n} \partial_{j}\left(a_{i j} \partial_{i} \psi_{R}\right) d x d \tau \\
& \leq C R^{-2} \int_{s}^{t} \int_{B_{2 R} \backslash B_{R}}|u|^{q}|x|^{2} d x d \tau+C R^{-1} \int_{s}^{t} \int_{B_{2 R} \backslash B_{R}}|u|^{q}|x| d x d \tau \\
& \leq C \int_{s}^{t}\|u(\cdot, \tau)\|_{L^{q}\left(B_{2 R} \backslash B_{R}\right)}^{q} d \tau \\
& \rightarrow 0 \quad(R \rightarrow \infty)
\end{aligned}
$$

and

$$
\begin{aligned}
\int_{s}^{t} \int_{\mathbb{R}^{n}} h(u) \mathbf{b} \cdot \nabla \psi_{R} d x d \tau & \leq C R^{-1} \int_{s}^{t} \int_{B_{2 R} \backslash B_{R}}|u|^{q}|x| d x d \tau \\
& \leq C \int_{s}^{t}\|u(\cdot, \tau)\|_{L^{q}\left(B_{2 R} \backslash B_{R}\right)}^{q} d \tau \\
& \rightarrow 0 \quad(R \rightarrow \infty)
\end{aligned}
$$

Consequently, letting $R \rightarrow \infty$ in (3.1), we obtain

$$
\begin{aligned}
& \int_{\mathbb{R}^{n}}|u(x, t)|^{q} d x+q(q-1) \int_{s}^{t} \int_{\mathbb{R}^{n}}|u(x, \tau)|^{q-2} \sum_{i, j=1}^{n} a_{i j}(x, \tau) \partial_{i} u(x, \tau) \partial_{j} u(x, \tau) d x d \tau \\
& \quad+\int_{s}^{t} \int_{\mathbb{R}^{n}}(-\operatorname{div} \mathbf{b}(x, \tau)+q c(x, \tau))|u(x, \tau)|^{q} d x d \tau \\
& =\int_{\mathbb{R}^{n}}|u(x, s)|^{q} d x
\end{aligned}
$$

This completes the proof of Theorem 1.3 
Proof of Corollary 1.4. (i) Applying Theorem 1.3 with $s=0$ and using $u(x, 0)=0$, we have by the assumption (p-i) that

$$
\begin{aligned}
& \int_{\mathbb{R}^{n}}|u(x, t)|^{q} d x+q(q-1) \lambda \int_{0}^{t} \int_{\mathbb{R}^{n}}|u(x, \tau)|^{q-2}|\nabla u(x, \tau)|^{2} d x d \tau \\
& \quad+\int_{0}^{t} \int_{\mathbb{R}^{n}}(-\operatorname{div} \mathbf{b}(x, \tau)+q c(x, \tau))|u(x, \tau)|^{q} d x d \tau \\
& \leq 0
\end{aligned}
$$

for all $t \in[0, T)$. Noting $-\operatorname{div} \mathbf{b}+q c \geq 0$ by the assumption (p-iii), we conclude $u(x, t)=0$ for $(x, t) \in \mathbb{R}^{n} \times[0, T)$.

(ii) Let $I=(-\infty, 0)$. Similarly to the above (i), applying Theorem 1.3 for $s<t<0$, we have by the assumption (p-iii) that

$$
\|u(t)\|_{L^{q}} \leq\|u(s)\|_{L^{q}} .
$$

Since $u \in L^{q}\left(\mathbb{R}^{n} \times(-\infty, 0)\right)$, there exists a sequence $\left\{s_{n}\right\}_{n=1}^{\infty} \subset(-\infty, 0)$ such that $\lim _{n \rightarrow \infty} s_{n}=-\infty$ and $\lim _{n \rightarrow \infty}\left\|u\left(s_{n}\right)\right\|_{L^{q}}=0$. Therefore, taking $s=s_{n}$ in (3.2) and then letting $n \rightarrow \infty$, we have $\|u(t)\|_{L^{q}}=0$. Since $t \in(-\infty, 0)$ is arbitrary, we conclude that $u \equiv 0$ on $\mathbb{R}^{n} \times(-\infty, 0)$. This proves Corollary [1.4.

\section{ACKNowledgement}

This work was supported by JSPS Grant-in-Aid for Scientific Research(S) Grant Number JP16H06339.

\section{REFERENCES}

[1] Amick, J.C., On Leray's problem of steady Navier-Stokes flow past a body in the plane. Acta Math 161 (1988), 71-130.

[2] Bildhauer, M., Fuchs, M., Zhang, G., Liouville-type theorems for steady flows of degenerate power law fluids in the plane. J. Math. Fluid Mech. 15 (2013), 583-616.

[3] Chat, D., Liouville type theorems for the Euler and the Navier-Stokes equations, Advances in Mathematics 228 (2011), 2855-2868.

[4] Chat, D., Liouville-Type theorems for the forced Euler equations and the Navier-Stokes equations. Commun. Math. Phys. 326, (2014), 37-48.

[5] ChaE, D., Remarks on the Liouville type problem in the stationary 3D Navier-Stokes equations. arXiv:1502.04793v1.

[6] Chat, D., Wolf, J., On Liouville type theorems for the steady Navier-Stokes equations in $\mathbb{R}^{3}$, J. Differential Equations 261 (2016), 5541-5560.

[7] Giga, Y., A remark on a Liouville problem with boundary for the Stokes and the Navier-Stokes equations, Discrete Contin. Dyn. Syst. Ser. S 6 (2013), 1277-1289.

[8] Gilbarg, D., Weinberger, H. F., Asymptotic properties of steady plane solutions of the Navier-Stokes equations with bounded Dirichlet integral. Ann. Scuola Norm. Sup. Pisa Cl. Sci. (4) 5 (1978), 381-404.

[9] Chamorro, D., Jarrín, O., Lemarié-Rieusset, P.-G., Some Liouville theorems for stationary Navier-Stokes equations in Lebesgue and Morrey spaces arXiv:180603003v1.

[10] Koch, G., Nadirashvili, N., Seregin, G., Sverak, V., Liouville theorems for the NavierStokes equations and applications. Acta Math. 203 (2009), 83-105.

[11] Korobkov, M.V., Pileckas, K., Russo, R., On the steady Navier-Stokes equations in $2 D$ exterior domains. http://arxiv.org/abs/1711.02400

[12] Korobkov, M.V., Pileckas, K., Russo, R., On convergence of arbitrary D-solution of steady Navier-Stokes system in 2D exterior domains. Arch. Rational Mech. Anal. 233 (2019), $385-407$.

[13] Kozono, H., Terasawa, Y., Wakasugi, Y., A remark on Liouville-type theorems for the stationary Navier-Stokes equations in three space dimensions. J. Funct. Anal. 272 (2017), 804-818. 
[14] Kozono, H., Terasawa, Y., Wakasugi, Y., Asymptotic properties of steady and nonsteady solutions to the $2 d$ Navier-Stokes equations with finite generalized Dirichlet integral. arXiv:1903.09969v3.

[15] Leray, J., Étude de diverses équations intégrals non linéaires et de quelques problèmes que pose l'Hydrodynamique. J. Math. Pures Appl. 12, 1-82 (1933).

[16] Seregin, G., Liouville type theorem for stationary Navier-Stokes equations, Nonlinearity 29 (2016), 2191-2195.

[17] Seregin, G., Silvestre, L., Šverák, V., Zlatoš, A., On divergence-free drifts, J. Differential Equations 252 (2012), 505-540.

[18] Souplet, P., Zhang, QI S., Sharp Gradient Estimate and Yau's Liouville Theorem for the Heat Equation on Noncompact Manifolds, Bulletin of the London Mathematical Society 38 (2016), 1045-1053.

(H. Kozono) Department of Mathematics, Faculty of Science and Engineering, Waseda University, Tokyo 169-8555, Japan, Research Alliance Center of Mathematical Sciences, Tohoku University, Sendai 980-8578, Japan

E-mail address, H. Kozono: kozono@waseda.jp, hideo.kozono.c7@tohoku.ac.jp

(Y. Terasawa) Graduate School of Mathematics, Nagoya University, Furocho Chikusaku NAGOYA 464-8602, JAPAN

E-mail address, Y. Terasawa: yutaka@math.nagoya-u.ac.jp

(Y. Wakasugi) Graduate School of Engineering, Hiroshima University, Higashi-Hiroshima, 739-8527, JAPAN

E-mail address, Y. Wakasugi: wakasugi@hiroshima-u.ac.jp 